\title{
Comparison between Multiple doses and Single-dose Steroids in Preventing the Incidence of Reintubation after Extubation among Critically ill Patients: A Network Meta- analysis
}

\author{
Chiwon Ahn \\ Chung-Ang University \\ Min Kyun $\mathrm{Na}$ \\ Hanyang University \\ Tae Ho Lim \\ Hanyang University \\ Bo-Hyoung Jang \\ Kyung Hee University \\ Wonhee Kim \\ Hallym University \\ Youngsuk Cho \\ Hallym University \\ Hyungoo Shin \\ Hanyang University \\ Jae Guk Kim \\ Hallym University \\ Juncheol Lee \\ Armed Forces Capital Hospital \\ Kyu-Sun Choi ( $\sim$ vertex-09@hanmail.net ) \\ Hanyang University
}

\section{Research Article}

Keywords: Steroid, Planned extubation, Reintubation, Network meta-analysis

Posted Date: January 29th, 2021

DOI: https://doi.org/10.21203/rs.3.rs-151255/v1

License: (c) (i) This work is licensed under a Creative Commons Attribution 4.0 International License. Read Full License 


\section{Abstract}

This study aimed to determine the frequency of prophylactic steroid administration to prevent reintubation after extubation in critically ill patients. We systematically searched MEDLINE, Embase, and Cochrane Library for studies regarding the preventive use of multiple doses or single-dose steroids prior to extubation on July 2020, and conducted a network meta-analysis (NMA) to compare these interventions. To assess the risk of bias of each included study, version 2 of the Cochrane risk-of-bias tool for randomized trials was used. Nine randomized control trials comprising 2,098 patients with comparisons of the three interventions were included. Use of multiple doses and single doses showed a significantly lower rate of reintubation compared with placebo (odds ratio [OR]: 0.43, 95\% confidence interval [CI]: 0.25-0.72; OR: 0.31, 95\% Cl: 0.14-0.69). However, the comparison between multiple doses and single doses showed no significant differences (OR: 1.22, 95\% Cl: 0.32-4.74). According to the surface under the cumulative ranking curve statistic, the treatments should be ranked as follows: single dose (87.1\%), high dose $(62.8 \%)$, and placebo $(0.1 \%)$. This NMA showed that the multiple doses were not statistically superior to the single dose in lowering the incidence of reintubation after extubation in critically ill patients. Therefore, use of a single-dose steroid can reduce the incidence of reintubation.

\section{Introduction}

Reintubation after extubation failure causes complications such as cardiovascular failure or ventilator-associated pneumonia, and is associated with an increased mortality rate ${ }^{1-3}$. Extubation should not be considered as a simple reversed process of intubation; the need for extubation should be planned on the day that the patient was intubated ${ }^{4,5}$. Even if the patients' medical condition is stable and no potential complications occurred, such as postextubation stridor, laryngeal edema, or reintubation, the need for extubation should be carefully planned ${ }^{4,6}$. The current guideline suggests that the prophylactic use of steroids prior to extubation is recommended as it is effective in reducing inflammatory airway edema, which can cause direct airway injury ${ }^{4,7}$. A recent meta-analysis also showed that the use of steroids is effective in reducing post-extubation stridor and reducing the incidence of reintubation after extubation ${ }^{8}$.

The difficult airway society guidelines suggest that $100 \mathrm{mg}$ of hydrocortisone (HC) should be administered every 6 hours ${ }^{4}$. In addition, Lin et al. (2016) showed that the administration of multiple doses is effective in reducing postextubation airway obstruction than the use of a single dose ${ }^{9}$. However, despite the suggested steroid dose and administration method, various steroid regimens have been used in previous studies. The diversity in the steroid dose and administration methods used in previous studies has unsettled practicing clinicians.

We performed a network meta-analysis (NMA), which allows a coherent analysis of all randomized controlled trials (RCTs), in order to evaluate the association between the administration of prophylactic steroids and the reintubation rate after extubation in intubated critically ill patients. Additionally, we analyzed the efficacy of various steroid doses (multiple doses, single dose, and placebo), by integrating all available direct and indirect evidence in the NMA.

\section{Results}

\section{Study selection}

After the online database search, 1,914 relevant articles were found in MEDLINE, 1,844 in Embase, and 1,871 in the Cochrane Library; meanwhile, two additional articles were found by manual searching. A total of 3,786 studies were 
identified after removal of duplicates, and 65 potentially relevant articles were retrieved after a full-text review (Fig. 1). The final nine RCTs selected met the eligibility criteria and included 2,098 patients ${ }^{9-17}$.

\section{Study characteristics}

Of the six RCTs that were published in East Asia, two were published in France (Table 1). The steroids used for treatment were methylprednisolone (MP), dexamethasone (DM), and HC. Regarding the methods of steroid administration, four studies used multiple doses, three studies used a single dose, and two studies used both single and multiple doses. With regard to the use of multiple doses or single dose, the amount to be administered once was an HC equivalent dose of 100-250 mg. In Lin et al.'s study (2016), the two different amounts of steroids were used in one comparison because both were administrated in multiple doses ${ }^{9}$.

Table 1

Baseline characteristics of included studies

\begin{tabular}{|c|c|c|c|c|c|c|c|c|}
\hline Author & $\begin{array}{l}\text { Year of } \\
\text { publication }\end{array}$ & Region & Period & $\begin{array}{l}\text { Population } \\
\text { in meta- } \\
\text { analysis }\end{array}$ & Age & Steroid & $\begin{array}{l}\text { Dose } \\
\text { frequency }\end{array}$ & $\begin{array}{l}\text { Equivalent dose } \\
\text { of } \\
\text { hydrocortisone }\end{array}$ \\
\hline $\begin{array}{l}\text { Darmon } \\
\text { et al. }\end{array}$ & 1992 & France & $\begin{array}{l}1986- \\
1987\end{array}$ & 664 & 53.16 & DM & Single & 200 \\
\hline Ho et al. & 1996 & Taiwan & 1990 & 77 & 62 & $\mathrm{HC}$ & Single & 100 \\
\hline $\begin{array}{l}\text { Cheng } \\
\text { et al. }\end{array}$ & 2006 & Taiwan & $\begin{array}{l}2002- \\
2004\end{array}$ & 128 & 66.12 & MP & $\begin{array}{l}\text { Multiple } \\
\text { or Single }\end{array}$ & 800 or 200 \\
\hline $\begin{array}{l}\text { Francois } \\
\text { et al. }\end{array}$ & 2007 & France & $\begin{array}{l}2001- \\
2002\end{array}$ & 698 & 66 & MP & Multiple & 400 \\
\hline $\begin{array}{l}\text { Lee et } \\
\text { al. }\end{array}$ & 2007 & Taiwan & $\begin{array}{l}2004- \\
2006\end{array}$ & 80 & 72.55 & DM & Multiple & 500 \\
\hline $\begin{array}{l}\text { Baloch } \\
\text { et al. }\end{array}$ & 2010 & Pakistan & $\begin{array}{l}2006- \\
2008\end{array}$ & 92 & 39.65 & DM & Multiple & 500 \\
\hline $\begin{array}{l}\text { Cheng } \\
\text { et al. }\end{array}$ & 2011 & Taiwan & $\begin{array}{l}2005- \\
2006\end{array}$ & 71 & 60.49 & MP & Single & 200 \\
\hline Yu et al. & 2014 & China & $\begin{array}{l}2010- \\
2013\end{array}$ & 162 & 67 & DM & $\begin{array}{l}\text { Multiple } \\
\text { or Single }\end{array}$ & 250 or 125 \\
\hline Lin et al. & 2016 & Taiwan & $\begin{array}{l}2007- \\
2010\end{array}$ & 126 & 74.09 & DM & Multiple & 1,000 or 500 \\
\hline \multicolumn{9}{|c|}{ MP, methylprednisolone; DM, dexamethasone; HC, hydrocortisone; NR, not reported } \\
\hline${ }^{\mathrm{a}} 1 \mathrm{mg} \mathrm{N}$ & $\mathrm{maHC}$ & $\mathrm{DM}=2$ & $\mathrm{HC}$ & & & & & \\
\hline
\end{tabular}

\section{Risk of bias}

With regard to the overall risk of bias for each included study, five studies had a low risk of bias, while four studies had some risk of bias. In the detailed assessment of all subcategories, the "risk of bias arising from the randomization process" of four studies were evaluated as some concerns risk of bias because the detailed descriptions of the randomization process were omitted or the baseline difference between intervention and control was shown in supplementary Fig. S1. 


\section{Quality of evidence}

We assessed the quality of evidence from each comparison for reintubation using the GRADEpro Guideline Development Tool. The GRADEpro tool revealed a high level of evidence between multiple doses and placebo, and a high level of evidence between single doses and placebo. However, the tool showed a moderate level between multiple doses and placebo due to imprecision (Table 2, Supplementary Table S1).

Table 2

Meta-analysis results for pairwise comparisons of steroid administration frequency

\begin{tabular}{|llllll|}
\hline Tx1 & Tx2 & No. of studies & $\mathrm{I}^{2}$ & OR (95\% Cl) & GRADE \\
\hline Multiple doses & Placebo & 6 & $0 \%$ & $0.43(0.25-0.72)$ & High \\
\hline Single dose & Placebo & 5 & $0 \%$ & $0.31(0.14-0.69)$ & High \\
\hline Multiple doses & Single dose & 2 & $0 \%$ & $1.22(0.32-4.74)$ & Moderate \\
\hline OR, odds ratio & & & & \\
\hline a Quality of evidence for direct estimate rated down by one level for serious imprecision \\
\hline
\end{tabular}

\section{Main analysis: Pairwise meta-analysis}

Each comparison included multiple doses, single dose, and placebo. There were six direct comparisons for multiple doses and placebo, five for single dose and placebo, and two for multiple doses and a single dose (Table 2, Supplementary Fig. S2). The multiple doses showed a significantly lower rate of reintubation compared with placebo (odds ratio [OR]: $0.43,95 \%$ confidence interval [CI]: $0.25-0.72$ ), and the single dose showed a significantly lower rate of reintubation compared with placebo (OR: $0.31,95 \% \mathrm{Cl}: 0.14-0.69)$. However, the comparison between multiple doses and single doses showed no significant differences (OR: 1.22, 95\% Cl: 0.32-4.74) (Table 2, Supplementary Fig. S3). There was no significant heterogeneity among the included studies within each comparison.

\section{Main analysis: Network meta-analysis}

Figure 2 shows the forest plot of the overall comparison. The inconsistency test at the global and local levels indicated no significant inconsistency (global level: $\mathrm{P}=0.9942$; local level: $\mathrm{P}=0.995,0.686$, and 0.824; Supplementary Fig. S4); there is no problem with accepting the consistency model. Indirect evidence showed that multiple doses or a single dose likely decreased the reintubation rate compared with placebo (Table 3). According to the surface under the cumulative ranking curve (SUCRA) statistic, which calculated the probability of each treatment, the treatment should be ranked in the following order: single dose (87.1\%), multiple doses (62.8 \%), and placebo $(0.1 \%)$ (Table 4$)$. Results of the statistical analysis showed that the single dose is superior to the multiple doses in terms of treatment effect. 
Table 3

League table for networks according to steroid administration frequency

\begin{tabular}{|llll|}
\hline & Placebo & Multiple doses & Single dose \\
\hline Placebo & - & $0.42(0.25-0.69)$ & $0.31(0.14-0.67)$ \\
\hline Multiple doses & $2.41(1.44-4.03)$ & - & $0.75(0.31-1.79)$ \\
\hline Single dose & $3.21(1.49-6.92)$ & $1.33(0.56-3.20)$ & - \\
\hline
\end{tabular}

Table 4

Rank probability and surface under the cumulative ranking (SUCRA) curve result

\begin{tabular}{|llllll|}
\hline Rank & $\mathbf{1}$ & $\mathbf{2}$ & $\mathbf{3}$ & Mean Rank & SUCRA \\
\hline Single dose & 0.741 & 0.257 & 0.002 & 1.3 & 0.871 \\
\hline Multiple doses & 0.259 & 0.741 & 0.001 & 1.7 & 0.628 \\
\hline Placebo & 0.000 & 0.003 & 0.998 & 3.0 & 0.001 \\
\hline
\end{tabular}

\section{Publication bias}

To assess for publication bias, we created a funnel plot (Supplementary Fig. S5). Overall, the studies showed 95\% Cls. In addition, the plot appeared symmetrical in shape.

\section{Discussion}

This NMA indicated the necessity of administering steroids in multiple doses or in a single dose to prevent the risk of reintubation after extubation in critically ill patients, and assessed the outcome-specific certainty of evidence using the Grading of Recommendations, Assessment, Development and Evaluation (GRADE) system compared with previous meta-analysis. The pairwise meta-analysis showed that there was no significant difference between multiple doses and single-dose steroids in terms of reducing the risk of reintubation; however, the NMA showed that the use of a single dose was statistically superior to the use of multiple doses. Additionally, both multiple doses and single-dose steroids have shown significant superiority in lowering the reintubation rate compared with placebo in several studies.

The results of this study were inconsistent with those of previous studies, which reported that multiple doses are more effective than single-dose steroids ${ }^{4,9}$. The duration of action of steroids is at least 8 hours; MP, an intermediate-acting steroid, 12-36 hours; and DM, a long-acting steroid, 36-72 hours ${ }^{18}$. In the included studies, multiple doses were administered every 4-6 hours. Considering the decrease in body distribution over time and the increase of drug concentration in the blood, multiple doses are a relatively high dose, and the duration of action is longer than that of a single dose. However, the evidence that multiple doses of steroids can lower reintubation than single doses remained unclear. The dosage of steroids and duration of use are important independent risk factors for the occurrence of side effects ${ }^{19}$. If multiple doses of steroids do not significantly lower the occurrence of reintubation compared with a single dose, this dosing method should not be used as it is cumbersome, is time consuming, and requires more manpower.

The steroids used before extubation are short acting and are administered in smaller doses; to date, no study has reported the direct side effects of steroid use during extubation. Even if side effects occur, they are reversible and 
more likely to be the effect of prolonged use of steroids in critically ill patients rather than the side effect of the use of prophylactic steroids. Therefore, clinicians might consider the use of steroids to prevent the occurrence of complications after extubation based on the patient's condition and environment.

The last study included in the analysis was conducted in 20169; no RCT has reported the use of steroids to prevent reintubation after extubation. In recent years, noninvasive ventilation (NIV) and high-flow oxygen therapy (HFOT) after extubation to prevent reintubation are used as alternative respiratory supports ${ }^{20-22}$. The latest NMA reported that NIV is the most effective respiratory support method for preventing reintubation after extubation ${ }^{23}$. None of the studies included in this meta-analysis used alternative respiratory support after extubation. Steroids used prior to planned extubation and NIV and HFOT used after extubation are not opposite treatments. Although no previous RCTs have used steroids and alternative respiratory support sequentially, these treatment methods can prevent the incidence of reintubation; however, further research is needed to confirm this finding.

This study has several limitations. First, the use of three types of steroids can cause bias. Although a standard steroid dose equivalent to the $\mathrm{HC}$ dose has been established to achieve the relative anti-inflammatory effect, the interpretation of results is limited due to the differences in the duration of action, time of onset, and duration of potency for each steroid. Second, a slightly direct comparison was performed between multiple doses and single dose. In the network statistics, statistical analysis was performed by adding the values of direct and indirect comparisons; statistically, the consistency was satisfied in this study. However, due to the small number of studies, the results cannot be considered as clinically significant. Hence, additional NMA should be conducted in future studies. Third, the included studies were conducted in China and Taiwan, except for one study, and the generalizability of the results was limited because the studies were only focused on a certain ethnic group and regional area.

In conclusion, this NMA showed that multiple doses of prophylactic steroids were not statistically superior to singledose steroids in reducing the incidence of reintubation after extubation among critically ill patients, and a single dose of steroid might be sufficient to reduce the incidence of reintubation. In addition, both methods are more effective than placebo. Although multiple doses of steroids can increase the steroid concentration and retention time in blood, its effect in preventing reintubation is not superior to that of a single dose. Therefore, a single dose can be used to reduce the rate of reintubation.

\section{Methods}

This NMA was performed in accordance with the Preferred Reporting Items for Systematic Reviews and MetaAnalyses statement for reporting network meta-analyses ${ }^{24}$.

\section{Search strategy}

MEDLINE, Embase, and Cochrane Library were systematically searched by two independent reviewers (Ahn C and $\mathrm{Na} \mathrm{MK)} \mathrm{for} \mathrm{studies} \mathrm{regarding} \mathrm{the} \mathrm{preventive} \mathrm{use} \mathrm{of} \mathrm{multiple} \mathrm{doses} \mathrm{or} \mathrm{single-dose} \mathrm{steroids} \mathrm{prior} \mathrm{to} \mathrm{extubation} \mathrm{in}$ critically ill medical patients from inception through July 2020. The detailed search strategy is presented in Supplementary Table S2. The reference lists of previous relevant studies and review articles were manually searched to identify other relevant literature.

\section{Study selection}


After excluding duplicate records, two reviewers (Ahn $\mathrm{C}$ and $\mathrm{Na} \mathrm{MK}$ ) independently screened the title and abstract of all selected articles to assess for eligible studies. During the preliminary screening, the full text of eligible articles was reviewed to determine whether these studies met the inclusion or exclusion criteria. No restrictions were imposed on the study period or type of steroid. A third reviewer participated in the discussion to adjudicate disagreements.

\section{Inclusion and exclusion criteria}

(1) Studies conducted in adult critically ill medical patients (age $\geq 18$ years) admitted in the intensive care unit who used steroids to prevent complications after extubation, (2) studies that reported the outcomes: reintubation, and (3) prospective RCTs were included in the analysis.

Meanwhile, (1) non-RCTs, including reviews, cohort studies, and crossover studies; (2) studies conducted in postsurgical patients; (3) studies that enrolled patients who underwent an unplanned extubation; (4) studies that did not report the outcome of interest; and (5) conference abstracts without full-text manuscripts were excluded.

\section{Groups of comparison: multiple doses vs single dose vs placebo}

The multiple dose group referred to patients who received two or more steroid injections on a regular interval prior to extubation. The single-dose group referred to patients who received one steroid injection. The placebo group referred to patients who did not receive steroid injections.

\section{Data extraction}

Two reviewers (Ahn $\mathrm{C}$ and Na MK) independently reviewed the full text of each included study and extracted the data using a standardized form. The abstracted data included the name of the author, publication year, duration of study, setting of the study, sample size, details of the population enrolled, type of steroid used, interval of steroid administration, and equivalent dose of $\mathrm{HC}$ that can achieve a relative anti-inflammatory effect [1 mg MP=5 mg HC, $1 \mathrm{mg} \mathrm{DM}=25 \mathrm{mg} \mathrm{HC}$. When discrepancies occur between reviewers, a discussion was made to reach a consensus.

\section{Quality assessment}

For the quality assessment of the included studies, version 2 of the Cochrane risk-of-bias tool for randomized trials (RoB 2) was used ${ }^{25}$. RoB 2.0 was divided into the following categories: "risk of bias arising from the interventions," "risk of bias due to missing outcome data," "risk of bias in measurement of the outcome," and "risk of bias in selection of the reported result." Each subcategory was rated as follows: "yes," "probably yes," "no," "probably no," and "no information." Next, using the evaluation result of each subcategory, the risk of bias was evaluated as low, high, or some concerns according to the evaluation algorithm suggested in RoB 2.0. Finally, the risk of bias was determined as low, high, or some concerns according to the overall quality evaluation criteria presented in RoB 2.0. Disagreements between the two reviewers were resolved by discussion.

\section{Statistical analysis}

The random effects NMA was performed using a frequentist framework to calculate the ORs for dichotomous outcomes and the corresponding $95 \% \mathrm{Cls}$. All statistical analyses were performed using the netmeta package in Stata 13.0 (Stata-Corp, College Station, TX, USA). A two-sided P value of less than 0.05 was considered significant. The homogeneity and consistency assumptions underlie the validity of evidence from NMA ${ }^{26}$. The inconsistencies between direct and indirect estimates in the entire network for each outcome were assessed locally with a loopspecific approach and globally with a design-by-treatment interaction model ${ }^{27}$. The treatment effects of various 
respiratory support methods were ranked according to the probabilities of leading to the best results based on the SUCRA for each outcome ${ }^{28}$. The SCURA value ranged from 0-100\%; a higher SUCRA value indicated the effectiveness of the method ${ }^{28}$. Each comparison was conducted using GRADE analysis (GRADEpro Guideline Development Tool, McMaster University, USA). Finally, we inspected the funnel plot for presence of publication bias using the netfunnel package in Stata 13.0.

\section{Declarations}

\section{Data availability}

The datasets generated during the current study are available from the corresponding author on reasonable request.

\section{Acknowledgements}

This research was supported by the Chung-Ang University Research Grants in 2021.

This work was supported by the research fund of Hanyang University (HY-201800000000589).

\section{Author contributions}

C.A. and K-S.C. designed the study and performed the data analysis. C.A. and M.K.N. performed the study selection and data extraction. C.A., M.K.N. and K-S.C. conducted the quality assessment and evidence rating. C.A. performed the database search and drafted the manuscript. All authors have read, revised, and approved the final manuscript.

\section{Competing interests}

The authors declare that there is no conflict of interests regarding the publication of this paper.

\section{References}

1. Frutos-Vivar, F. et al. Outcome of reintubated patients after scheduled extubation. J. Crit. Care 26, 502-9. https://10.1016/j.jcrc.2010.12.015. (2011).

2. Peterson, G. N. et al. Management of the difficult airway: a closed claims analysis. Anesthesiology 103, 33-9. https://10.1097/00000542-200507000-00009. (2005).

3. Epstein, S. K. Extubation failure: an outcome to be avoided. Crit. Care 8, 310-2. https://10.1186/cc2927. (2004).

4. Difficult Airway Society Extubation Guidelines Group. Difficult Airway Society Guidelines for the management of tracheal extubation. Anaesthesia 67, 318-40. https://10.1111/j.1365-2044.2012.07075.x. (2012).

5. Higgs, A. et al. Guidelines for the management of tracheal intubation in critically ill adults. Br. J. Anaesth. 120, 323-52. https://10.1016/j.bja.2017.10.021. (2018).

6. Boles, J-M. et al. Weaning from mechanical ventilation. Eur. Respir. J. 29, 1033-56. https://10.1183/09031936.00010206. (2007).

7. Schmidt, G. A. et al. Official Executive Summary of an American Thoracic Society/American College of Chest Physicians Clinical Practice Guideline: Liberation from Mechanical Ventilation in Critically III Adults. Am. J. 
Respir. Crit. Care Med. 195, 115-9. https://10.1164/rccm.201610-2076ST. (2017).

8. Kuriyama, A., Umakoshi, N., \& Sun, R. Prophylactic corticosteroids for prevention of postextubation stridor and reintubation in adults: a systematic review and meta-analysis. Chest 151, 1002-10.

https://10.1016/j.chest.2017.02.017. (2017).

9. Lin, C-Y., Cheng, K-H., Kou, L-K., \& Lee, C-H. Comparison of high-and low-dose dexamethasone for preventing postextubation airway obstruction in adults: a prospective, randomized, double blind, placebo-controlled study. Int. J. Gerontol. 10, 11-6. https://doi.org/10.1016/j.ijge.2015.10.002. (2016).

10. Hutton, B. et al. The PRISMA extension statement for reporting of systematic reviews incorporating network meta-analyses of health care interventions: checklist and explanations. Ann. Intern. Med. 162, 777-84. https://10.7326/M14-2385. (2015).

11. Sterne, J. A. C. et al. RoB 2: a revised tool for assessing risk of bias in randomised trials. BMJ. $366, \mid 4898$. https://10.1136/bmj.I4898. (2019).

12. Donegan, S., Williamson, P., D'Alessandro, U., \& Smith, C. T. Assessing key assumptions of network metaanalysis: a review of methods. Res. Synth. Methods 4, 291-323. https://10.1002/jrsm.1085. (2013).

13. Higgins, J. P. et al. Consistency and inconsistency in network meta-analysis: concepts and models for multi-arm studies. Res. Synth. Methods 3, 98-110. https://10.1002/jrsm.1044. (2012).

14. Salanti, G., Ades, A. E., \& loannidis, J. P. Graphical methods and numerical summaries for presenting results from multiple-treatment meta-analysis: an overview and tutorial. J. Clin. Epidemiol. 64, 163-71. https://10.1016/j.jclinepi.2010.03.016. (2010).

15. Darmon, J. Y. et al. Evaluation of risk factors for laryngeal edema after tracheal extubation in adults and its prevention by dexamethasone. A placebo-controlled, double-blind, multicenter study. Anesthesiology 77, $245-51$. https://10.1097/00000542-199208000-00004. (1992).

16. Ho, L. I. et al. Postextubation laryngeal edema in adults. Risk factor evaluation and prevention by hydrocortisone. Intensive Care Med. 22, 933-6. https://10.1007/BF02044118. (1996).

17. Cheng, K. C. et al. Intravenous injection of methylprednisolone reduces the incidence of postextubation stridor in intensive care unit patients. Crit. Care Med. 34, 1345-50. https://10.1097/01.CCM.0000214678.92134.BD. (2006).

18. François, B. et al. 12-h pretreatment with methylprednisolone versus placebo for prevention of postextubation laryngeal oedema: a randomised double-blind trial. Lancet 369, 1083-9. https://10.1016/S01406736(07)60526-1. (2007).

19. Lee, C. H., Peng, M. J., \& Wu, C. L. Dexamethasone to prevent postextubation airway obstruction in adults: a prospective, randomized, double-blind, placebo-controlled study. Crit. Care 11, R72. https://10.1186/cc5957. (2007).

20. Baloch, R., Jakhrani, N., Lal, A., \& Mehmood, N. Role of dexamethasone for prevention of post-extubation airway obstruction in critically ill adult patients. J. Surg. Pak. 15, 3-8. (2010).

21. Cheng, K. C. et al. Methylprednisolone reduces the rates of postextubation stridor and reintubation associated with attenuated cytokine responses in critically ill patients. Minerva Anestesiol. 77, 503-9. (2011).

22. Yu, Y. et al. Use of dexamethasone for preventing postextubation airway obstruction in adults: a prospective randomized study. J. Intern. Med. Concepts Pract. 9, 134-7. (2014).

23. Schimmer, B. P., \& Funder, J. W. ACTH, adrenal steroids, and pharmacology of the adrenal cortex. Goodman and Gilman's The pharmacological basis of therapeutics 12th ed (McGraw-Hill, New York, 2011). 
24. Yasir, M., Goyal, A., Bansal, P., \& Sonthalia, S. Corticosteroid adverse effects. (Treasure Island (FL): StatPearls Publishing, 2020)

25. Vargas, F. et al. Intermittent noninvasive ventilation after extubation in patients with chronic respiratory disorders: a multicenter randomized controlled trial (VHYPER). Intensive Care Med. 43, 1626-36. https://10.1007/s00134-017-4785-1. (2017).

26. Fernandez, R. et al. High-flow nasal cannula to prevent postextubation respiratory failure in high-risk nonhypercapnic patients: a randomized multicenter trial. Ann. Intensive Care 7, 47. https://10.1186/s13613-0170270-9. (2017).

27. Hernández, G. et al. Effect of postextubation high-flow nasal cannula vs conventional oxygen therapy on reintubation in low-risk patients: a randomized clinical trial. JAMA. 315, 1354-61. https://10.1001/jama.2016.2711. (2016).

28. Zhou, X. Preventive use of respiratory support after scheduled extubation in critically ill medical patients-a network meta-analysis of randomized controlled trials. Crit. Care 24, 370. https://10.1186/s13054-020-03090-3. (2020).

\section{Figures}
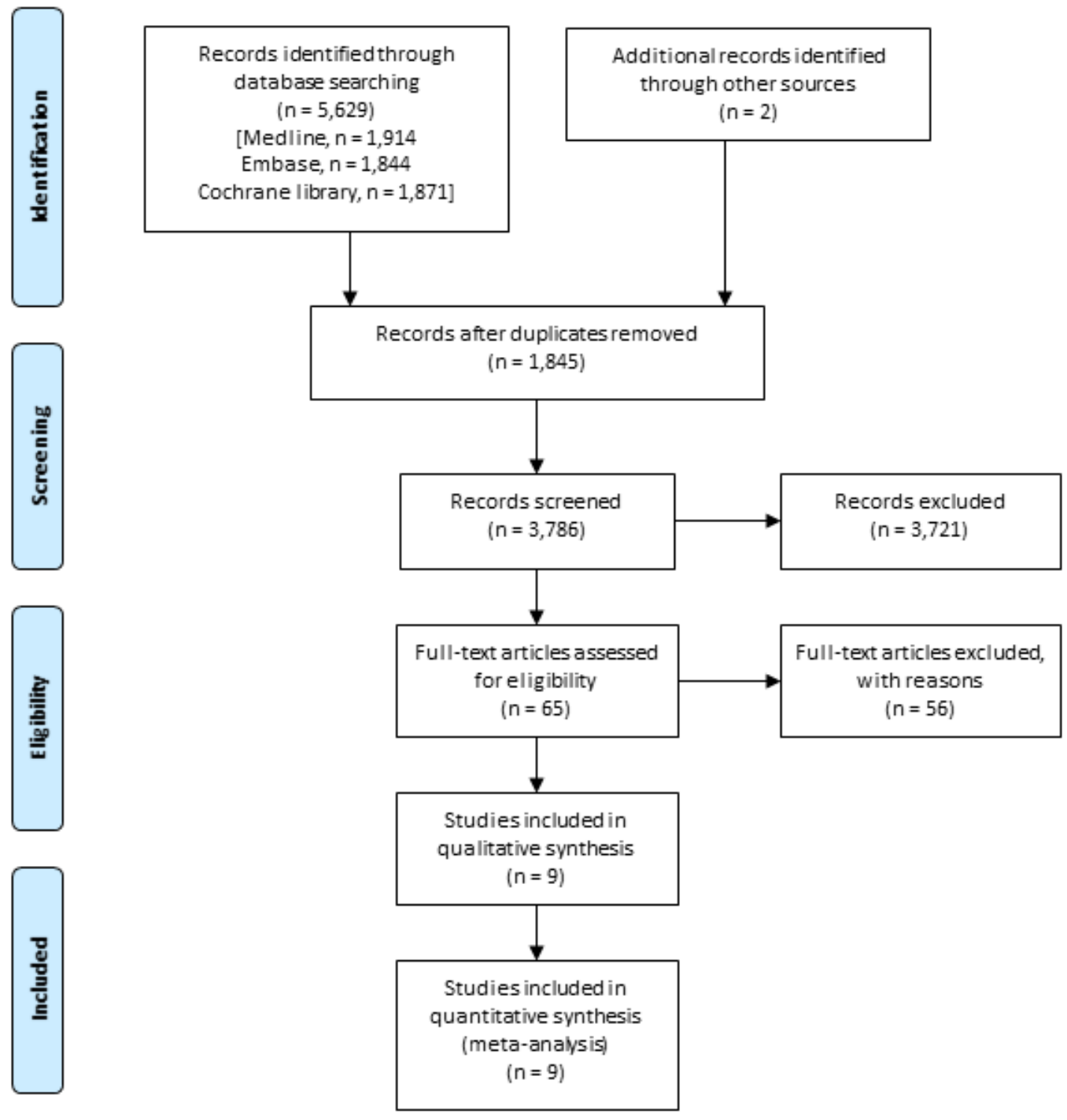
Figure 1

PRISMA flowchart of the study selection process

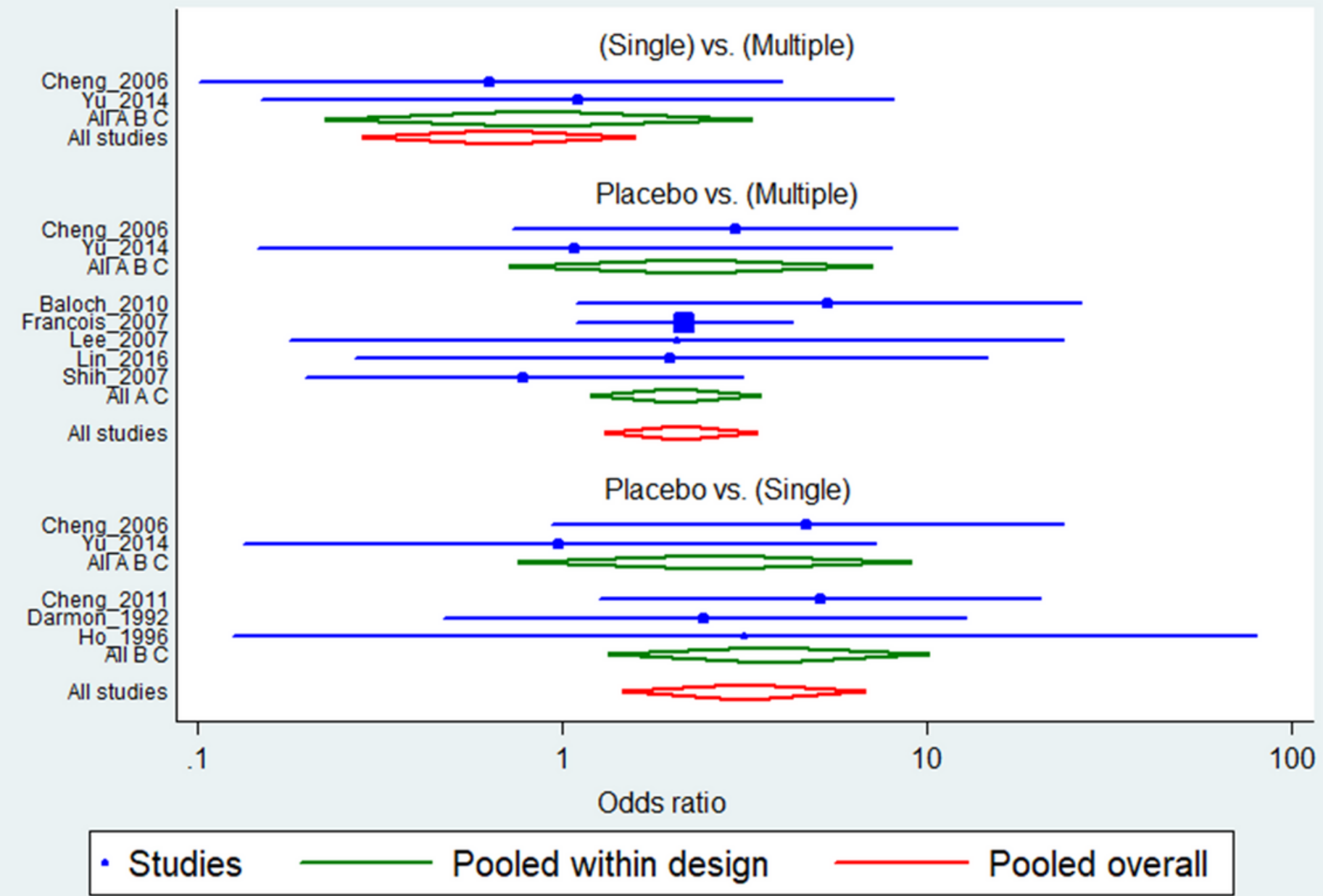

Test of consistency: chi2(2) $=0.25, P=0.884$

Figure 2

Forest plot of the network meta-analysis for reintubation rate

\section{Supplementary Files}

This is a list of supplementary files associated with this preprint. Click to download.

- PRISMA2009checklist.pdf

- supplementaryfile.docx 